\title{
Advantages of lateral BMD DEXA measure- ment and concomitant lateral spinal imaging at the point of service over conventional PA measurement in the estimation of BMD
}

\section{Paul Sneider MB BCh, DMR (D), RCP\&S (Engl) \\ Parktown North Mammography and Bone Density Centre Johannesburg}

osteopaenic patients and in 26\% who had osteoporosis with lateral IVA assessment. It was therefore concluded that both of these modalities were useful and made a greater contribution in the assessment of bone density loss, therefore enabling a more appropriate treatment protocol to be instituted and also improving patient compliance. The latter occurred because patients would rather accept a visual image of their illness such as a spine fracture or BMD value visually displayed on a graph than a verbal explanation for their asymptomatic but serious disease.

\section{Introduction}

Galen (AD 130 - 200) described the skeleton 'as poles to tents and walls to houses, so are bones to living creatures, for other features naturally take form from them and change them' The date of the earliest recorded use of the term osteoporosis in English, as far as can be determined, was in 1846 according to Webster's Ninth
Collegiate Dictionary.

Osteoporosis is an important disease because it is associated with significant morbidity and mortality. Therefore its accurate diagnosis is important in view of its prevalence and the dire consequences when inadequately treated. It is generally accepted that osteoporosis affects up to $30 \%$ of the female population. As it is symptomless and the first time a patient may become aware of it is when a fracture occurs, it behoves all physicians not only to diagnose this condition, but to educate their patients so that adequate treatment can be given effectively. In 1991 Ross et $a .^{2}$ clearly enunciated the importance of vertebral fractures in patients with bone loss. In their study they were able to show that even with a high bone mineral density (BMD) the presence of one vertebral fracture would increase the future vertebral fracture risk by 10.2 times, and this would increase to 75 times when there were multiple spine fractures with a low BMD. In 1993 Genant et al. ${ }^{3}$, realising the importance of spinal fractures, classified them not only according to severity by grading them but also morphologically as wedge deformities, biconcave deformities and crush deformities and published a most elegant chart (Fig. 1) depicting these deformities. This was important because many examinations for osteoporosis are conducted by nonradiologists who may be unfamiliar with the morphological appearance of spinal fractures, particularly when they are subtle (early). It has been estimated that about $25 \%$ of American women over the age of 50 years have vertebral fractures and this percentage increases dramatically with advancing age, so that by the age of 75 , more 


\section{ORIGINAL ARTICLE}

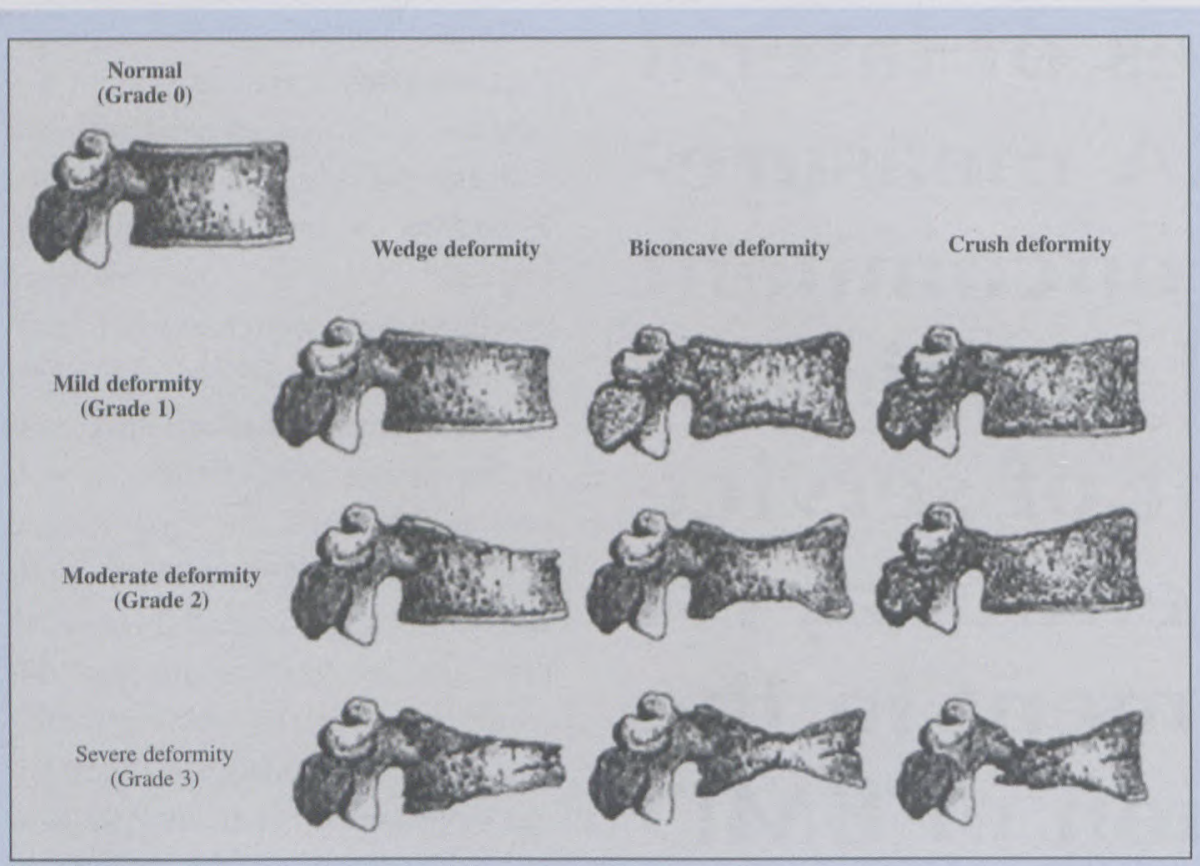

Fig. 1. The Genant classification of fracture grade

than $33 \%$ of women will have at least one vertebral fracture..$^{3.6}$ As many of these fractures are asymptomatic, they are difficult to diagnose clinically and may require imaging for their recognition. Loss of height is unreliable and carries a low sensitivity for vertebral fractures. With modern fan beam technology, high-resolution lateral spinal images are now available at the point of service. During a DEXA examination, such images are available using an exposure time of only 10 seconds and utilising $1 / 100^{\text {th }}$ of the conventional X-ray dose. This technology is now available in the Hologic Delphi range of DEXA fan beam scanners and is called instant vertebral assessment (IVA). Together with this facility lateral BMD measurement of the upper lumbar spine is also currently available.

The aim of this study was to assess the value of lateral spine BMD measurements and compare them with conventional PA measurements, and also to see if lateral spine imaging
(IVA) which is available as part of the DEXA scanner would be of additional value.

\section{Method}

The apparatus used was a Hologic Delphi fan beam whole body DEXA scanner. The patients were all adult females, sequentially selected. After informed consent, they were all examined in the supine and lateral decubitus positions (PA and lateral). The lumbar spine was evaluated for total (L1 - L4) BMD and individual vertebral BMDs. Lateral measurements of $\mathrm{L} 2$ and L3 were then evaluated for the total and individual BMDs. ' $T$ ' scores were obtained from the large Hologic database. Both hips were also examined in the supine position with the hips in internal rotation and their ' $\mathrm{T}$ ' scores were computed from the NHANES 111 database.? The hip studies were not used for assessment as part of this study. Patients with hip prostheses were excluded and forearm DEXA measurements were not included in this study. The WHO classification $^{8}$ for ' $T$ ' scoring was used for the diagnosis. A normal examination is when the ' $\mathrm{T}$ ' score is $<1.0$ standard deviation (SD) below the peak bone mass at 30 years of age. When $\mathrm{T}=1-2.5$ SDs below the peak, this was taken to represent osteopaenia. With $\mathrm{T}>2.5 \mathrm{SDs}$ below the peak, osteoporosis was diagnosed.

\section{Results}

The total number $(N)$ of patients was 144 . Their ages varied between 20 and 81 years, with a mean age of 53.4 years.

Of the total number of cases $(N=$ $144)$, the following diagnoses were made using the criteria enunciated above.

Therefore, (Tables I and II) the total number of cases requiring modification of their treatment protocols increased by 51 cases out of 144 (35\%), which is in keeping with most studies.

\begin{tabular}{|c|c|}
\hline Osteopaenia $(N)$ & Osteoporosis $(N)$ \\
\hline Total $=113$ & 62 \\
\hline
\end{tabular}

$\begin{gathered}\text { Table II. Total abnormal PA lumbar } \\
\text { spinal BMD (L1 - L } 4)\end{gathered}$
\begin{tabular}{cc} 
Osteopaenia $(N)$ & Osteoporosis $(N)$ \\
\hline 49 & 13 \\
Total $=62$ & \\
\hline
\end{tabular}

As can be seen from Table III, 30 patients $(21 \%)$ who would have been reported as having normal BMD measurements in the PA position had osteopaenia as seen in the lateral DEXA spine evaluation. Similarly, 13 


\section{ORIGINAL ARTICLE}

\begin{tabular}{l}
$\begin{array}{l}\text { Table III. Normal report by PA DEXA } \\
\text { BMD total lumbar spine (L1 - LA) } \\
\text { study only converted after total lateral }\end{array}$ \\
BMD (L2 - L3) DEXA examinaiton to: \\
\hline \\
\hline
\end{tabular}

patients (9\%) were shown to be osteoporotic with lateral lumbar spine BMD measurement which was evaluated in the PA position as normal.

Table IV helps to evaluate the usefulness of IVA where high-quality lateral and PA views of the dorso-lumbar spine are obtained during the DEXA examination. Four patients had unexplained asymptomatic vertebral fractures. Fourteen patients (10\%) with

\begin{tabular}{|lcc|}
\hline $\begin{array}{c}\text { Table IV. IVA studies showing verte- } \\
\text { bral fractures* }\end{array}$ \\
\hline & $N$ & $\%$ \\
\hline Osteopaenia & 14 & 10 \\
Osteoporosis & 37 & 26 \\
Normal & 4 & $<1 \%$ \\
All patients were post-menopausal. & \\
\hline
\end{tabular}

BMD values indicating osteopaenia had asymptomatic fractures and, similarly, $37(26 \%)$ of the osteoporotic patients had asymptomatic spinal fractures.

\section{Typical case histories}

\section{Mrs A. D. Age 50 (Fig. 2)}

Routine examination revealed the following:
Total BMD in PA direction, 1.087 $\mathrm{g} / \mathrm{cm}^{2}$. 'T' value +0.40 .

Total BMD in lateral position 0.640 $\mathrm{g} / \mathrm{cm}^{2}$.' $\mathrm{T}$ ' value -2.5 .

Osteoprosis, therefore, could only be diagnosed because of the lateral BMD study.

\section{Mrs M. P. Age 70 (Fig. 3)}

Complaint: backache.

Total BMD in PA direction 0.827 $\mathrm{g} / \mathrm{cm}^{2}$. 'T' value -2.30 .

Total BMD in lateral position 0.598 $\mathrm{g} / \mathrm{cm}^{2}$. 'T' value -3.00 .

Despite having severe osteoporosis and multiple vertebral fractures seen with IVA, osteoporosis as per the WHO classification could only be diagnosed with lateral BMD and IVA. IVA shows the vertebral column status.

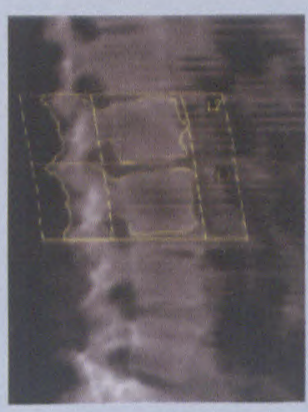

$113 \times 73$

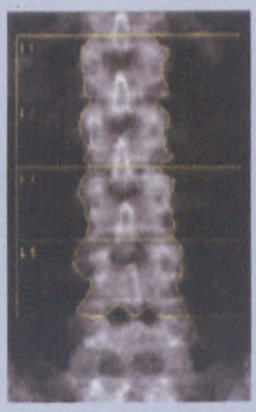

$116 \times 146$

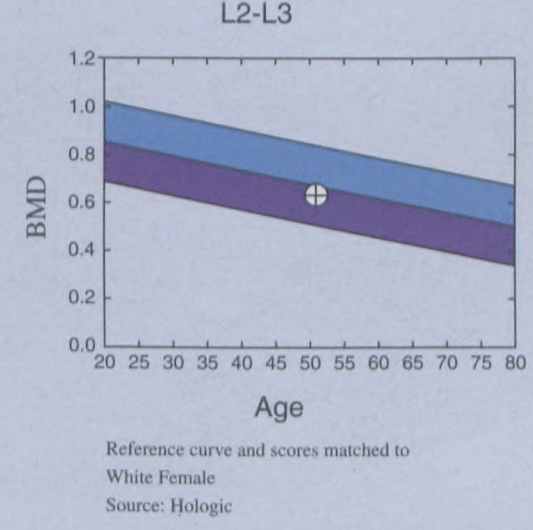

L1-L4

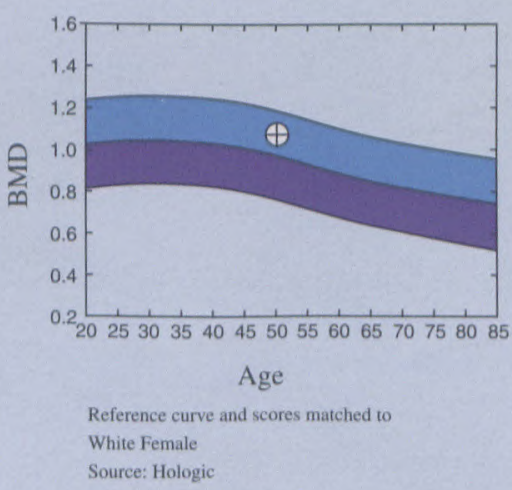

Scan Date: 05 March 2003 - A0305030E

\section{DXA Results Summary:}

\begin{tabular}{|cccc|}
\hline Region & BMD & T - & Z - \\
& $\left(\mathrm{g} / \mathrm{cm}^{2}\right)$ & Score & Score \\
L2-L3 & 0.640 & -2.5 & -0.4 \\
\hline
\end{tabular}

Total BMD CV $2.6 \%$

WHO Classification: Osteoporosis

Fracture Risk: High

Scan Date: 05 March 2003 - A0305030D

\begin{tabular}{|c|c|c|c|}
\hline Region & $\begin{array}{l}\text { BMD } \\
\left(\mathrm{g} / \mathrm{cm}^{2}\right)\end{array}$ & $\begin{array}{c}\text { T - } \\
\text { Score }\end{array}$ & $\begin{array}{c}\text { Z- } \\
\text { Score }\end{array}$ \\
\hline L1-L4 & 1.087 & 0.4 & 1.2 \\
\hline
\end{tabular}

Total BMD CV $1.0 \%$

WHO Classification: Normal

Fracture Risk: Not Increased

Fig. 2. Mrs $A D$, Lateral spine $B M D$ measurement finds the osteoporosis. 


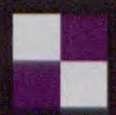

Diagnostios

Gadopentetate dimeglumine
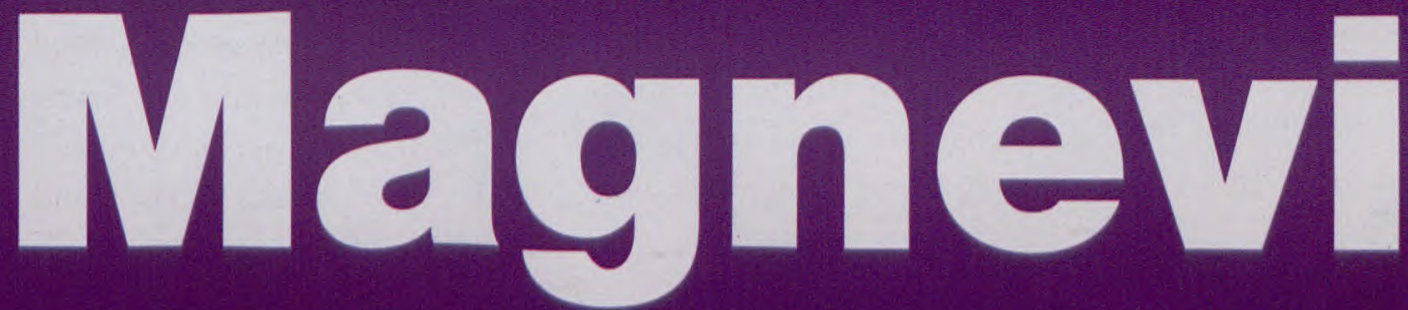

(B)

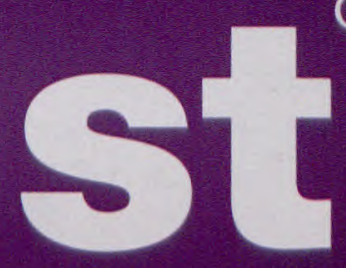




\section{ORIGINAL ARTICLE}

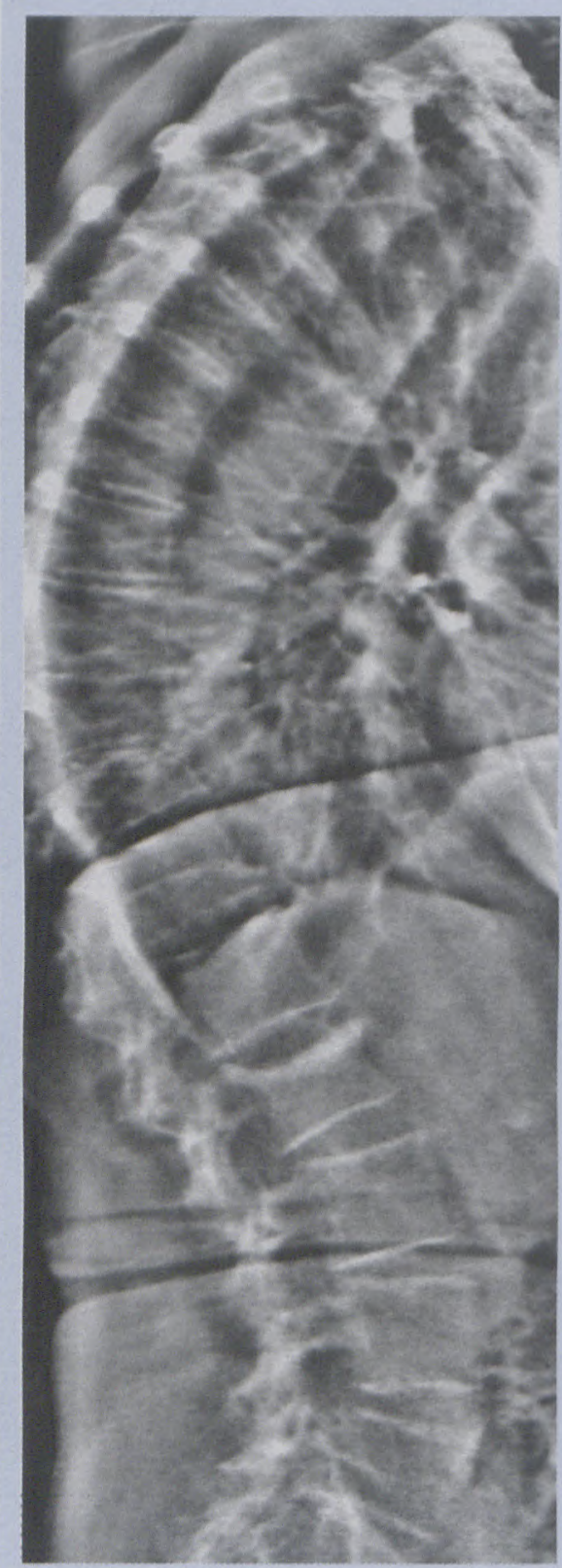

Fig. $3 a$ and b. IVA showing multiple spine fractures.

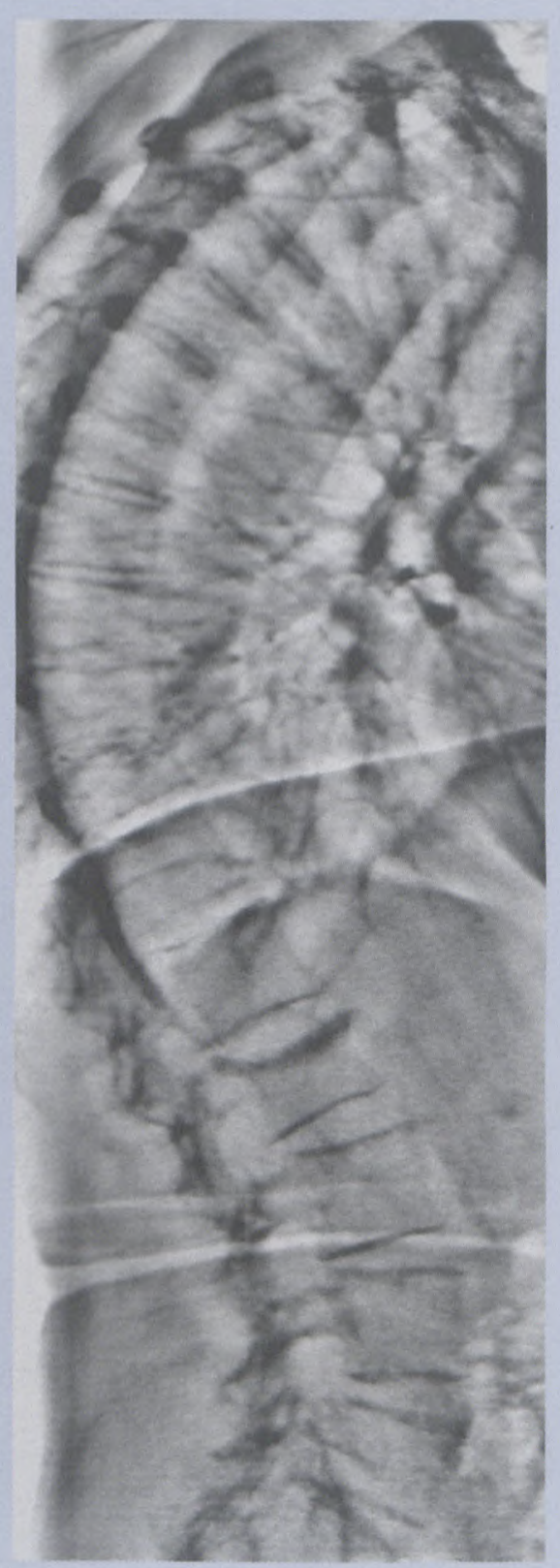

\section{Discussion}

This study confirms others that stress the importance of morphologic spinal assessment in the evaluation of bone loss so that appropriate therapy can be given. ${ }^{2,39-11}$ A significant number of patients in this series would have had inadequate therapeutic protocols without spine studies that demonstrate vertebral fractures. In ment available, confirming Faulkner et al.'s ${ }^{13}$ study of the discordance in BMD values depending on the method of examination. Purely as a result of lateral lumbar spinal BMD measurement, $35 \%$ of our cases were upgraded in terms of severity of bone loss. Also of importance is the fact that $10 \%$ of the osteopaenic patients and $26 \%$ diagnosed as osteoporotic had vertebral fragility fractures. Four postmenopausal women with normal BMDs, however had unexplained vertebral fractures with no history of previous trauma. IVA morphological assessment of the dorso-lumbar spine produces excellent images that can be computer-manipulated, zoomed, exposure-corrected and black/white reversed. They offer an excellent alternative to conventional X-ray studies. Because osteoporosis is a 'silent' disease with little or no symptomatology in the absence of a fracture, patient compliance is often difficult to obtain. ${ }^{14}$ It has also been estimated that only about $7 \%$ of American women with osteoporosis get treatment and about $75 \%$ of women over the age of menopause have never discussed this problem with their medical advisors.?

Approximately $20 \%$ of women over the age of 50 years have vertebral fractures, ${ }^{4}$ which are asymptomatic and clinically difficult to detect. A modern DEXA scanner such as the Hologic Delphi uses fan beam technology and produces high-quality lateral studies of the dorso-lateral spine. It has also been found that if patients can be shown the effects of osteoporosis (including the 'dowager's hump'), compliance improves as a result of better understanding by asymptomatic patients of the complicating issue of fractures. ${ }^{14}$ Genant and his 


\section{ORIGINAL ARTICLE}

coworkers $^{3}$ have published a classification of fracture involvement in the vertebral column with three grades of severity. This is a valuable contribution, as it results in an easily explained and recognisable way to report, categorise and follow up vertebral fractures. Having this facility at the point of service allows a high-quality study of the vertebral column to be made easily, rapidly, and at no additional cost with a low radiation dose. Therefore, an additional conventional X-ray examination can be avoided thus saving time, money, high radiation dose and inconvenience. The economic and health impact of the complications of osteoporosis are unacceptably high. It has been estimated that there will be approximately 800000 hip fractures in the USA by the year 2050 and in Asia that number is expected to rise to 3250000 . $^{15}$ Approximately $20 \%$ of these patients die in the acute phase of this disease, and of the remainder about $50 \%$ will never achieve full mobility again. In 1999 , approximately US $\$ 23000000000$ was spent in the USA alone on the treatment of osteoporotic fractures (National Osteoporosis Foundation of the USA). ${ }^{15}$ This study confirms the findings of Faulkner et al. ${ }^{13}$ when discussing the discordance of patient classification using ' $\mathrm{T}$ ' scores. They were able to demonstrate that with conventional PA DEXA spine examinations the mean $-2.50 \mathrm{SD}$ level (osteoporosis) occurred at between 75 and 85 years, whereas with lateral spine BMD evaluation osteoporosis was already seen on average by the age of 62 years, which was similar to that found using quantitative cat scanning (QCT). Although accurate for the measurement of cancellous bone content, the latter examination, however, is not really practical because of high costs, large radiation dose and difficulty in obtaining adequate precision for comparison studies and follow up. As a result of this study, DEXA scanning for low bone mass, which includes IVA and lateral spine BMD measurement, indicates that significant numbers of patients will be reclassified because of the assessment of spinal fractures and the true BMD of cancellous bone in the vertebral column. This obviates falsely high vertebral PA BMDs because of osteophytes, sclerosis and aortic calcification in postmenopausal patients. Therefore, the appropriate treatment protocols can be applied at an earlier stage in these patients. Having these facilities at the point of service is useful and results in a cheaper, more efficient and convenient complete bone density loss evaluation, which is not only more accurate than conventional PA spinal BMD, but is also likely to improve patient compliance due to their better understanding of the relevant issues. ${ }^{14}$

\section{References}

1. Brown. JM. Osteoporosis, a growing problem (Editorial). J S C Med Assoc 2001; 97: 116.

2. Ross PD, Davis JW, Epstein RS, Wasnich RD. Pre-existing fractures and bone mass predict vertebral fracture incidence in women. Ann Intern Med 1991; 114: 919-923.

3. Genant HK, Wu CY, van Kujik C, Nevitt MC. Vertebral fracture assessment using a semiquantitative technique. J Bone Miner Res 1993; 8: 1137-1148.

4. Gordon L, Pope TL, Monen S. Value of vertebra X-rays in osteoporosis. I S C Med Assoc 2001; 97: 95-98.

5. Melton LJ, Atkinson EJ, Cooper C, et al. Prevalence and incidence of vertebral deformities. Osteoporos Int 1993; 3: 113-119.

6. Delmas PD, Ensrud KE, Adachi JD, et al., for the Multiple Outcomes of Raloxifene Evaluation (MORE) Investigators. MORE Trial. J Clin Endocrinol Metab 2002; 87: 3609-3617.

7. Third National Health and Nutrition Examination Survey (NHANES III). US Department of Commerce, 1996.

8. The World Health Organisation Study Group. Geneva: WHO, 1994: 129, 843.

9. Genant HK, Li J, Wu CY, et al. Vertebral fractures in osteoporosis. Journal of Clinical Densitometry 2000; 3: 280-290.

10. Rea JA, Chen M, Li J, et al. Visual vertebral deformity assessment by X-ray absorptiometry: a highly predictive method to rule out vertebra fractures. Bone 1998; 23(5): Suppl, S160.

11. Melton LJ 3rd Atkinson EJ, Cooper C, O'Fallon WM, Riggs BL. Vertebral fractures predict subsequent fractures. Osteoporos Int 1999; 10: 214 221

12. Finkelstein JS, Cleary RL, Butler IP, et al. A comparison of lateral versus antero-posterior spine dual energy X-ray absorptiometry for the diagnosis of osteoporosis. J Clin Endocrinol Metab 1994; 78: 724-730.

13. Faulkner KG, von Stetten E, Miller PJ. Discordance in patient classification using $\mathrm{T}$ scores. Journal of Clinical Densitometry 1999; 2: 343-350.

14. Silverman SL, Greenwald M, Klein RA, Drinkwater BL. Effect of bone density information on decisions about hormone replacement therapy: A randomized trial. Obstet Gynecol 1997; 89: 321-325.

15. National Osteoporosis Foundation. 1998 Guidelines For the Use of Bone Densitometry. Washington, DC: 1998. 